\title{
How can the use of lung ultrasound in cardiac arrest make ultrasound a holistic discipline. The example of the SESAME-protocol.
}

\section{Daniel A. Lichtenstein}

Service de Réanimation Médicale, Hôpital Ambroise-Paré, F-92100 Boulogne (Paris-Ouest), France

\begin{abstract}
The most critical application of critical ultrasound - cardiac arrest - is the opportunity for technical considerations. The necessity to immediately detect reversible causes is integrated in the concept of holistic ultrasound. Holistic ultrasound is defined as a discipline where each element interacts with the others, and where the understanding of each of them allows understanding the whole. A narrow machine (not necessarily a laptop), a fast start-on time, a simple keyboard highlighting three useful buttons, a universal microconvex probe able to immediately detect pneumothorax, then deep venous thrombosis, then abdominal bleeding, then pericardial tamponade, then cardiac anomalies will allow a fast protocol. The concept of holistic ultrasound is particularly on focus in the first step done at the lung (search for pneumothorax and clearance for fluid therapy), since the best image is obtained with the simplest equipment devoid of traditional facilities (image filtering, harmonics, time lag, Doppler...). The same simple gray-scale equipment is used for the other steps, all what is needed is to see the real-time image of what is facing the probe: the very principle of visual medicine. The same approach can be used with no change, just more quietly, for many less urgent settings.
\end{abstract}

Keywords: cardiac arrest, critical ultrasound, lung ultrasound, pneumothorax

It is familiar to use the word "disruptive" when physicians speak of the recent ultrasound craze. The credit of this revolution is usually given to technological progresses which have made machines smaller. The enthusiasm of the young generation which appropriates this visual tool is understandable. Using the example of the cardiac arrest, which will be extrapolated to multiple less dramatic settings, we will just show in this Review that older equipments were able to do this revolution, not only before - but also better. This article could have been written in 1982.

Any cardiac arrest of unknown cause raises a central question: is there a cause accessible to diagnosis, then therapy? Any tool able to expedite a diagnosis should be privileged. The usual gestures (within hospitals) include

Received 01.07.2014 Accepted 03.07.2014

Med Ultrason

2014, Vol. 16, No 3, 252-255

Corresponding author: Daniel A. Lichtenstein

Medical Intensive Care Unit

Hospital Ambroise-Paré

F-92100 Boulogne (Paris-West University)

France

E-mail: D.Licht@free.fr cardiac compressions, control of airway, massive oxygen... When all this is done, finding a cause is extremely difficult in these stressing settings. The physical examination is too limited. There is no time at all for any test... but ultrasound. The most critical application of critical ultrasound is found in cardiac arrest, when each second makes the prognosis.

\section{Technical considerations for optimizing a SESAME-protocol}

We describe our 1992 equipment, completely obsolete for those who see only its age. We use daily this providential machine. We don't look at its height. Not a laptop, it is $28-\mathrm{cm}$ high, not a problem since the trolley brings it at human distance for bedside work, and the ceilings are high enough. We look at its width, $32 \mathrm{~cm}$ with cart, which makes it one of the narrowest trolleybased machines in the world, a feature allowing optimal transportation at the bedside, in the I.C.U., E.R., O.R., all settings where each saved $\mathrm{cm}$ makes the difference. We don't look at its weight. The cathode ray tube, providing an analogical image quality (up to now the best 
resolution), is heavy, not a problem since its wheels allow easy transportation. Regarding the wheels, one can use the term of "disruptive technology", even if a 5000 years old discovery. Narrow design, wheels, the machine is immediately brought at the bedside without effort. Bedside ultrasound was possible using the excellent ADR-4000 with its 44-cm width (compare with today's laptops), a 1982 technology, our first unit.

The machine is switched-on. This takes 7 seconds. Seven seconds: an old machine, but the fastest of the world. With machines needing several minutes of starton time and unfortunately not ready, there is nothing to do but wait. Then we initiate the SESAME-protocol: the abbreviation of the long abbreviation SESAMOOSIC, for "Sequential Emergency Scanning Assessing Mechanism Or Origin of Shock of Indistinct Cause". [1]

The SESAME-protocol is a fast protocol used for extreme shock or just simple cardiac arrest. It is based on the causes which are the most frequent, the most easy to diagnose, the most easy to manage: pneumothorax, pericardial tamponade, abdominal bleeding, venous thrombosis (quite equivalent to pulmonary embolism). This immediately brings the dilemma: which probe should be used first? If we chose the bad probe, every second used for changing probes, setting etc., will be lost time.

In the SESAME-protocol, once the unit switchedon ( 7 seconds), we are exempted to make this difficult choice: we use one probe. Not the cardiac, not the vascular, not the abdominal probe. The probe we use (since 1992) is a microconvex one, with two outstanding properties: a $1-17 \mathrm{~cm}$ penetration, a resolution suitable for lung, heart, abdomen, veins... The term "universal" probe means that it has the best compromise for the whole body. Each specialized probe is perfect (or quite perfect) for its use (cardiac for the heart, etc.) but unsuitable for the other uses. Our probe is ideal for the lungs, all veins (from subclavian to caval), most of the abdomen, and suitable for the basic heart. Please beware, some familiar manufacturers propose "microconvex" probes, which have neither the resolution nor the penetration of ours, and are consequently not universal.

One probe: no time lost for choosing the best one. We really think that the major leaders of critical ultrasound should try it at least once for just making an opinion.

Not a futile advantage of the universal probe (apart from cost-savings), this philosophy saves precious centimeters: instead of these lateral racks (which make cumbersome machines), our machine, not a laptop, has a top where objects (probe) can be fixed, and remains slim laterally.

Another advantage, our simple keyboard which is flat (since 1982, allowing fast cleaning) has a few functions.
Inexperienced physicians facing cardiac arrest with some usual machines must master an impressive number of buttons in a haste.

Which setting? Having to choose means losing time. Since 1989, we have solved this problem by using the "no setting" by default, always ready: all filters have been withdrawn. This is critical for exploiting the potentials of lung ultrasound in the extreme emergencies. The technology must help, not confuse. An instant response is mandatory. Any time lag will confuse. A natural image allows analysis of lung artifacts (A-lines, lung rockets), of prime importance in critical settings. Harmonics etc can blur the artifacts. This was easy in the 1982 ADR-4000 and our 1992 unit, which had no filter. Today, it is difficult to find machines without filters. Some destructive filters cannot be by-passed. Although we begin to see in modern machines the word "Lung" in the settings, which is not bad, we are not far from a misconception, since we don't have a "lung" setting: the setting we use is the same at the whole body, the best compromise for expediting our fast examinations.

Now the SESAME-protocol can begin.

\section{Description of a SESAME-protocol}

The step-1 is devoted to the lung assessment. Cardiac arrest, lung first. Why? Because lung windows are everywhere, no fraction of second is lost for finding them. Because a highly reversible cause of cardiac arrest can be immediately ( $<3$ seconds) suspected: pneumothorax. This step- 1 is done while the compression operator stops the compression, keeps his hands on-site, ready to resume. It uses an anterior area located between upperBLUE-point and lower BLUE-point, adapted to the extreme emergency, assuming that a pneumothorax able to create a cardiac arrest is substantial. The intubation must have been done wisely, avoiding too generous meters of tube inserted within the thorax: in asystole, no lung pulse can be generated, preventing the diagnosis of one lung intubation. The user checks simultaneously the presence of lung sliding, and the absence of lung rockets, allowing for immediate, massive, fluid therapy.

Pneumothorax generates the A'-profile of the BLUEprotocol. Time is lacking for searching a lung point. We prefer, in the case of an A'-profile, make a manual percussion. The slightest asymmetry, associated with an A'profile in a suggestive setting (e.g., cardiac arrest here) legitimates the diagnosis of pneumothorax. Beginning by percuting the lung would be too chancy.

When a pneumothorax is ruled out, the Step 2-3 of the SESAME-protocol scans veins and abdomen. Veins first in nontraumatic arrest (searching for DVT), abdomen first in traumatic arrest (searching for free fluid, likely blood 
here). These targets illustrate the spirit of the SESAMEprotocol: although not direct signs, they clearly suggest pulmonary embolism or hypovolemia as the cause, in an appropriate context, i.e., here, just cardiac arrest.

Some doctors have learned to diagnose peritoneal fluid using the FAST-protocol, but one can also detect for a GI-tract bleeding. If this bleeding is nontraumatic, the word FAST shouldn't be used (since 1985, we have used the words "ultrasound search for free blood" for the same result: using first-line ultrasound). Several small areas of bleeding (each pleura, plus a little at the abdomen, plus one liter at one femur etc) can cause a cardiac arrest. Although this ultrasound travel may be time-consuming, note one main principle of holistic ultrasound: if an Aprofile has been detected at the Step-1 (the lung), giving massive fluid therapy is legitimate: the A-profile means a pulmonary artery occlusion pressure below $18-\mathrm{mm}-\mathrm{Hg}$, and fluid therapy is an urgent option allowing the cardiac resuscitation to be efficient - this data is available in the first seconds, before the cardiac step.

Critical ultrasound is a holistic discipline. Facing hypovolemic arrest, the fluid therapy is urgent. A large venous line must be inserted in an extreme haste. If devices for trauma (cervical collar, G-suit...) prevent to puncture the usual dirty sites, or for any other reason, our probe can be used without drawback at the subclavian area, especially because... it is not vascular. Vascular probes don't have the ergonomy suitable for infra-clavicular subclavian vein canulations, a procedure we routinely practice since 1989.

Critical ultrasound is a holistic discipline. If the cardiac arrest is due to a pulmonary embolism, the chance to find a proximal thrombosis is weak. Our equipment scans the calf, using an anterior approach, a transversal scanning, without Doppler. A negative test does not exclude the diagnosis, but makes it less likely (five times), since in the BLUE-protocol, this occurs in only $1 / 5$ th of cases.

If no bleeding nor DVT are found, the SESAMEprotocol comes to Step-4: the heart. The first attempt is done via subcostal route (with the same microconvex probe). Here is the permanent risk to face unsuitable cardiac windows. The compression operator discontinues the compressions, keeping hands on-site. The pericardial tamponade is now on focus. If no subcostal window is present after a few seconds, one must use the thoracic approach - hoping again for a window. We have nothing to add to the well-known and excellent protocols describing the echocardiographic patterns in cardiac arrest, between asystole and all cases of pulseless electric activity.

If no reversible cardiac cause has been found, one must resume the compressions. Here, the contact product we have created has a medical relevance, because the compressions can be done immediately after cardiac analysis. Unlike the traditional gel which makes a slippery landscape, unsuitable for efficient compressions (or need time-consuming removal of the gel), our product has chemical properties which make the skin surface nonslippery.

The SESAME-protocol has carefully suppressed all these unexpected limitations (some apparently futile) which can suddenly appear at any step of the management, potentialized by the extreme stress. Complex keyboards turn into hindrances to novices. Several probes make cables inextricably mixed. Cables lying on the floor favor the risk of a machine tip-over when suddenly mobilized (one imagines the triple consequence for the machine, the doctor's foot, the patient). Serious problems occur when each of these small difficulties is added to another, in addition to large laptop width, filters which alter the instant response etc.

\section{How can SESAME-protocol be extrapolated to less urgent settings}

Our 1992 machine is permanently configured "SESAME-protocol", because it is ready for the worst. Some famous leaders of critical ultrasound argued that cardiac arrest does not happen so often. Here, we are touching the deep definition of holistic ultrasound. The approach, settings etc, adapted when speed is life and each second has to be worked out, is used with no adaptation in our daily tasks (venous line insertion, thoracentesis, search for free blood...). And this can be extrapolated to countless more quiet settings in a dozen of disciplines.

The search for pneumothorax, life-saving here, will be done using the same rules, just more quietly, in all cases where the physician wants to check there is no pneumothorax. This regards anesthesiology, thoracic surgery, many others. Using a 7-seconds start-on time and a 32$\mathrm{cm}$ width machine, medical time will just be saved. The "no setting" setting provides the best resolution, the best instant response. With the unique probe and the flat keyboard, less time is spent for cleaning the probe/keyboard.

\section{How should one manage with current machines?}

Nowadays laptop machines are good. Each probe (cardiac, vascular, abdominal) is good for its devoted region of interest. Filters and computers allow sophisticated calculations, at the heart for example. It is possible to make any kind of ultrasound with any kind of equipment. The community must just accept the properties and limitations of these machines (in addition to the lateral volume, cost, absence of universal probe and other details). One must learn to swap the probes rapidly. The reader 
has, once again, understood that in cardiac arrest, the little benefit gained in sophisticated calculations that one has no time to do is balanced by the drawback to have a long start-on time, for dealing only with this drawback.

Regarding the future, we ask to the manufacturers to come back to previous technologies which worked better. Integrating the lung is the future of clinical ultrasound.

Trans-esophageal echocardiography during cardiac arrest management is a good, elegant idea, but a little long to implement for the whole planet. The SESAMEprotocol is a way to limit this costly alternative each time a cause has been found.

Regarding pocket machines, our first comment is that for hospital use, we have 32-cm width machines on carts, coming at appropriate height for working. Thanks to the cart, we can work with two hands (we need our two hands for making critical ultrasound) and have all equipments together. On the contrary, pocket machines can be precious in areas where space is really limited. In our airborne activity, in the choice of pocket machines now available, we use a gray-scale system without Doppler, with one probe design near to our microconvex probe, and a resolution allowing a universal use, at the heart, lung, venous and abdominal areas (whole body ultrasound), showing that it is possible to use yesterday's technology for a today's tool. For those hospital physicians wanting to have their own machine in hospitals, just as an alternative to our system (or because they don't like to share), we are not opposed to this concept. Realistic, we are quite sure that these machines will be bought by billions in the times to come.

A last word about Doppler, a facility we did not use in the SESAME-protocol. Emphasizing Doppler without mastering lung ultrasound is a misconception. When critical ultrasound will be completely routine, when the community will use its holistic power (i.e., e.g., replac- ing the absence of Doppler by direct data extracted from lung ultrasound: BLUE-protocol, FALLS-protocol), we will then study its potentials. Not before.

\section{Conclusions}

We have described how holistic ultrasound deals with cardiac arrest, and hope to have demonstrated that the disruptive revolution of critical ultrasound was not the fact of recent technologies, but more of scientifically assessments done before (using it is true suitable technologies). The current planetary revolution was possible, for cheap, 20 years before. The manufacturers should certainly be acknowledged, but also all doctors who use the tool for their daily practice (not to forget those who, not waiting the laptop revolution, used ultrasound before it was on fashion, jeopardizing their carrier for the improvement of mankind healthcare). In the light of the example of cardiac arrest management, ultrasound becomes holistic when the lung, its most vital component, is integrated, prioritized, adapted for use by several disciplines, not only critical care but pre-hospital medicine, pulmonology, internal medicine, etc., not to forget pediatrics, probably the most important.

\section{Acknowledgements}

Our sincere thanks to Petru Mircea and Radu Badea, for what they did for helping us and for their precious friendship.

\section{References}

1. SESAME-protocol. In: Lung Ultrasound in the Critically III. Springer-Verlag (in press) 